\title{
Helicity Amplitudes In the Hypercentral Constituent Quark Model
}

\author{
M.M. Giannini, E. Santopinto, A. Vassallo \\ Dipartimento di Fisica dell'Università di Genova, I.N.F.N. Sezione di Genova, Italy
}

October 9, 2018

\begin{abstract}
We report on the recent results of the hypercentral constituent quark model 1, 2]. The model contains a spin independent three-quark interaction which is inspired by QCD lattice calculations and reproduces the average energy values of the $S U(6)$ multiplets. The splittings are obtained with a residual generalized $S U(6)$-breaking interaction, including an isospin dependent term 2]. The long standing problem of the Roper resonance is absent and all the 3- and 4-star states are well reproduced. The model has also been used for predictions concerning the electromagnetic transition form factors giving a good description of the medium $Q^{2}$-behaviour 3 , 4. In particular the calculated $S_{11} A_{\frac{1}{2}}$ helicity amplitude agrees very well with the recent CLAS data [5]. Finally the ratio of the elastic form factors of the proton [6], calculated including kinematic relativistic corrections, exhibits a substantial decreasing with $Q^{2}$ in agreement with the recent TJNAF experiment [7] .
\end{abstract}

\section{The Hypercentral Model}

The model [1] consists of a hypercentral quark interaction containing a linear plus coulomb-like term as suggested by lattice QCD calculations [8]. It can be considered as the hypercentral approximation of the two-body potential or as a three-body potential

$$
V(x)=-\frac{\tau}{x}+\alpha x \quad, \quad \text { with } \quad x=\sqrt{\rho^{2}+\lambda^{2}},
$$

where $x$ is the hyperradius defined in terms of the Jacobi coordinates $\boldsymbol{\rho}$ and $\boldsymbol{\lambda}$. A hyperfine term of the standard form [9] is added and treated as a perturbation. After having fixed the quark mass $m$ to $1 / 3$ of the nucleon mass, the average energies of the $S U(6)$-multiplets are described with $\tau=$ 4.5 and $\alpha=1.61 \mathrm{fm}^{-2}$, while the strength of the hyperfine interaction is 
determined by the $\Delta$ - Nucleon mass difference. The wave functions of the various resonances are therefore completely determined (the few parameter of the model fixed once for all at the reproduction of the spectrum) and they have been used for the calculation of the photocouplings [3], the transition form factors to the negative parity resonances [4, the elastic form factors [10] and the ratio between the electric and magnetic form factors of the proton [6].

\section{Electromagnetic transition end elastic form factors}

The baryon spectrum is usually described quite well by various Constituent Quark Models 1, 9, 11, 12, although the various models are quite different. In order to distinguish among the various forms of quark dynamics one has to study in a consistent way all the physical observables of interest and not only the spectrum which is a static property. The helicity amplitudes for the electroexcitation of baryon resonances, are calculated using the states determined by the model and a non relativistic current for point quarks.

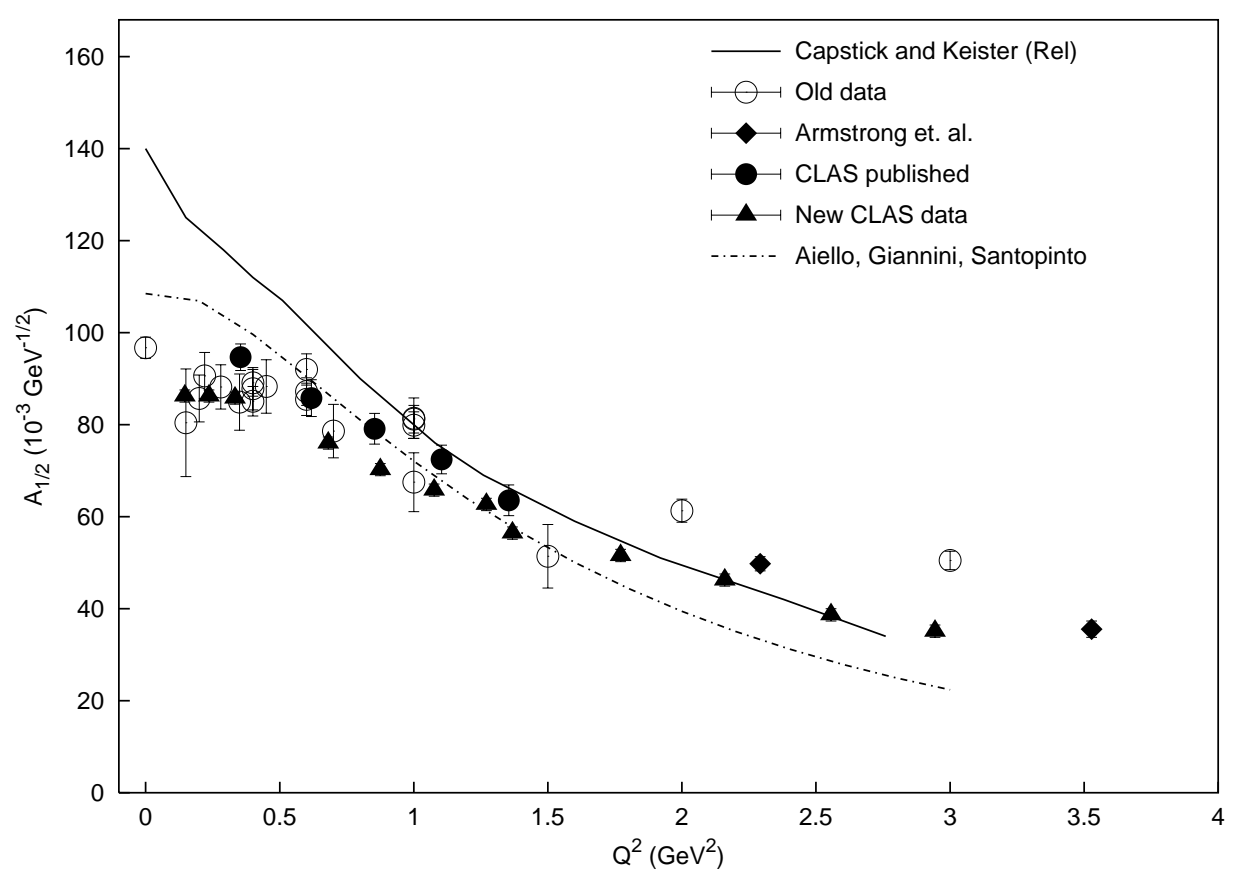

Figure 1: Comparison between the experimental data [5] for the helicity amplitude $A_{1 / 2}^{p}$ for the $S_{11}(1535)$ resonance and the calculations with the hCQM, lower curve [4] also compared with Capstick and Keister result, upper curve [13.

In Fig. 1 we report the helicity amplitude for the $S_{11}(1535)$ resonance. 
Similar results are obtained for the remaining negative parity nucleon resonances 4] and also in a systematic way for all the other 3-4 star and 1-2 star resonances [14].

In general the $Q^{2}$ behaviour is reproduced, except for discrepancies at small $Q^{2}$, especially in the $A_{3 / 2}^{p}$ amplitude of the transition to the $D_{13}(1520)$ state. These discrepancies could be ascribed to the non-relativistic character of the model, and to the lack of explicit quark-antiquark configurations which may be important at low $Q^{2}$. The kinematical relativistic corrections at the level of boosting the nucleon and the resonances states to a common frame are not responsible for these discrepances, as we have demonstrate in Ref. [15.

These boosts effects are on the contrary important for the elastic e.m. form factors. Taking into account the boosts of the 3-quark states for the nucleon from the rest frame to the Breit frame one can write

$$
G_{E}\left(Q^{2}\right)=F_{e l}^{C} G_{E}^{n r}(q / g), \quad G_{M}\left(Q^{2}\right)=F_{e l}^{M} G_{M}^{n r}(q / g),
$$

where $G_{E}^{n r}$, and $G_{M}^{n r}$ are the electric and magnetic form factors as given by the non relativistic quark model, $F_{e l}^{C}$ and $F_{e l}^{M}$ are kinematical factors and $g=E / M$. The formula of Eq.2 can be used for any CQMs [10, 15].In particular, the elastic form factors of Eq. (2), calculated using as input the nucleon form factors obtained in the hCQM, lead to an improvement of the theoretical description [10, 6, especially the shape of the elastic form factors as a function of $Q^{2}$ is similar to that of the experimental data. In Fig. 2 we report the ratio of the electric and magnetic form factors of the proton, $R=\mu_{p} \frac{G_{E}\left(Q^{2}\right)}{G_{M}\left(Q^{2}\right)}[6]$.

The data of a recent polarization transfer experiment at TJNAF show a significant deviation from the scaling behaviour, which is reproduced up to $1.5(\mathrm{GeV} / \mathrm{c})^{2}$ by the hCQM model calculation [6] (full curve in Fig. 2). It should be reminded that the non relativistic calculation gives $R=1$ and it remains 1 within $1 \%$ even if the hyperfine mixing is included. The decreasing of the ratio $R$ with $Q^{2}$ is due to the different behaviour of the relativistic correction for the electric and magnetic parts.

\section{Generalized SU(6)- breaking interac- tion}

There are different motivations for the introduction of a flavour dependent term in the three-quark interaction. The well known Guersey-Radicati mass formula 16 contains a flavour dependent term, which is essential for the description of the strange baryon spectrum. In the chiral Constituent Quark Model [12, 17], the non confining part of the potential is provided by the interaction with the Goldstone bosons, giving rise to a spin- and isospin-dependent part, which is crucial in this approach for the description of the lower part of the spectrum. More generally, one can expect that 


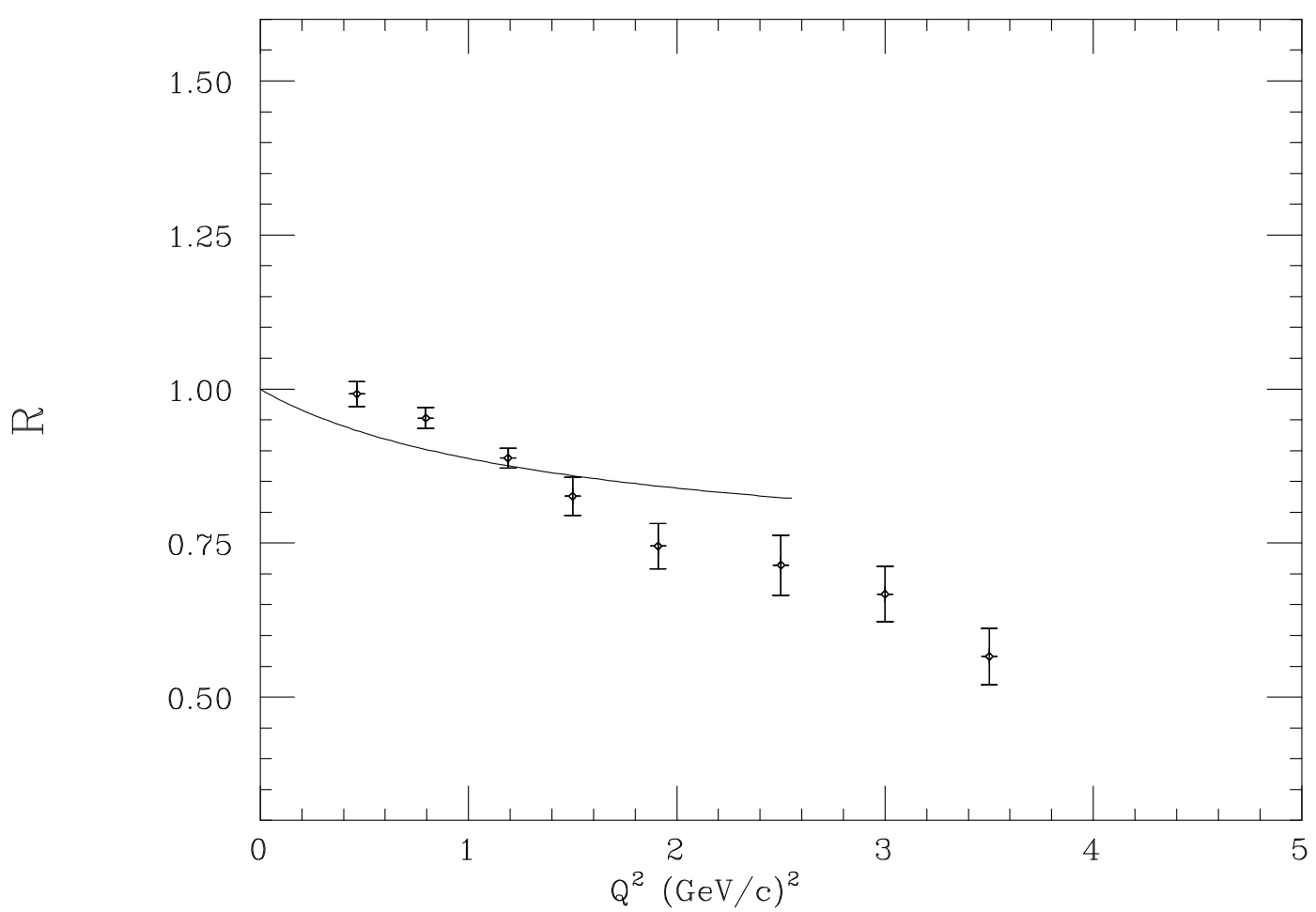

Figure 2: The ratio $R=\mu_{p} G_{E} / G_{M}$ calculated with the hCQM [6]. The points are the data from a recent TJNAF experiment [7]

the quark-antiquark pair production can lead to an effective residual quark interaction containing an isospin (flavour) dependent term. We have introduced isospin dependent terms in the hCQM hamiltonian. The complete interaction used is given by 2$]$

$$
H_{\text {int }}=V(x)+H_{\mathrm{S}}+H_{\mathrm{I}}+H_{\mathrm{SI}},
$$

where $V(x)$ is the linear plus hypercoulomb $\mathrm{SU}(6)$-invariant potential, while $H_{\mathrm{S}}+H_{\mathrm{I}}+H_{\mathrm{SI}}$ is a residual $\mathrm{SU}(6)$-breaking interaction, with $H_{\mathrm{S}}$ a smeared standard hyperfine term, $H_{\mathrm{S}}$ a spin dependent term, $H_{\mathrm{I}}$ isospin dependent and $H_{\mathrm{SI}}$ spin-isospin dependent.

The resulting spectrum for the $3 *^{*}$ and $4{ }^{*}$ - resonances is shown in Fig.3 [2]. The $N-\Delta$ mass difference is no more due only to the hyperfine interaction, which contribute about 35\%, while the remaining splitting comes from the spin-isospin term, (50\%), and from the isospin one, (15\%).

\section{Conclusions}

We have presented various results predicted by the hypercentral Constituent Quark Model compared with the experimental data. We have also 

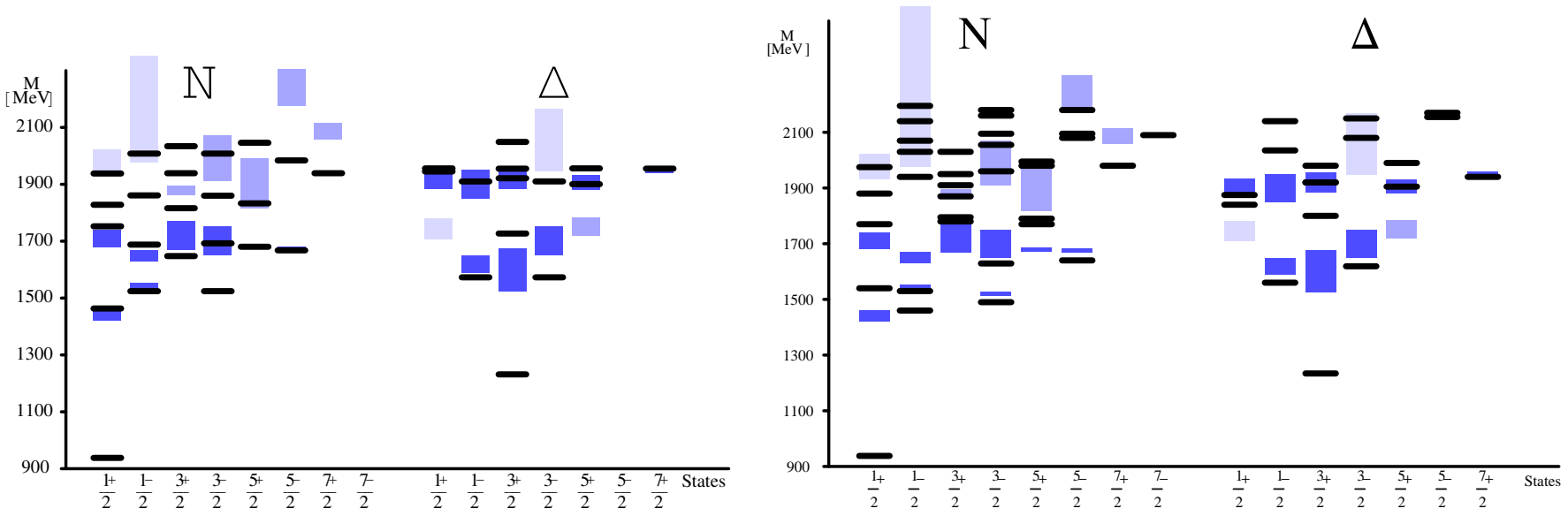

Figure 3: On the left the non strange spectrum obtained with the hCQM (complete interaction (3)). The shadowed boxes represent the experimental data from PDG with their uncertainty [18]: the dark grey boxes for the 3- and 4-star resonances and the light grey boxes for the 1and 2-stars. On the right are reported the results of the Isgur-Capstick model

shown that in the hCQM a flavour dependent potential can be introduced leading to improved splittings within the $S U(6)$-multiplets. A relativistic description of the dynamical properties of the nucleon is important and inevitable in particular for the electromagnetic form factors.

\section{References}

[1] M. Ferraris, M.M. Giannini, M. Pizzo, E. Santopinto and L. Tiator, Phys. Lett. B364, 231 (1995).

[2] M.M. Giannini, E. Santopinto and A. Vassallo, Eur. Phys. J. A12, 447 (2001); M.M. Giannini, E. Santopinto and A. Vassallo, Nucl. Phys. A699, 308(2002).

[3] M. Aiello, M. Ferraris, M.M. Giannini, M. Pizzo and E. Santopinto, Phys. Lett 387, 215 (1996).

[4] M. Aiello, M. M. Giannini, E. Santopinto, J. Phys. G: Nucl. Part. Phys. 24, 753 (1998)

[5] V. D. Burkert arXiv:hep-ph/0207149.

[6] M. De Sanctis, M.M. Giannini, L. Repetto, E. Santopinto, Phys. Rev. C62,025208 (2000).

[7] M.K. Jones et al., Phys. Rev. Lett. B84,1398 (2000).

[8] Gunnar S. Bali, Phys. Rep. 343, 1 (2001).

[9] N. Isgur and G. Karl, Phys. Rev. D18, 4187 (1978); D19, 2653 (1979); D20, 11 (1979); S. Godfrey and N. Isgur, Phys. Rev. D32, 189 (1985). 
[10] M. De Sanctis, E. Santopinto, M.M. Giannini, Eur. Phys. J. A1, 187 (1998).

[11] S. Capstick and N. Isgur, Phys. Rev. D 34,2809 (1986).

[12] L. Ya. Glozman and D.O. Riska, Phys. Rep. C268, 263 (1996).

[13] S. Capstick and B.D. Keister, Phys. Rev.D 51, 3598 (1995).

[14] M.M. Giannini, E.Santopinto, M. Aiello, to be published.

[15] M. De Sanctis, E. Santopinto, M.M. Giannini, Eur. Phys. J. A2, 403 (1998).

[16] F. Guersey and L.A. Radicati, Phys. Rev. Lett. 13, 173 (1964).

[17] L. Ya. Glozman, Z. Papp, W. Plessas, K. Varga, R. F. Wagenbrunn, Phys. Rev. C57, 3406 (1998).

[18] Particle Data Group, Eur. Phys. J. C15, 1 (2000). 